\title{
Disfluency patterns in Alzheimer's disease and Frontotemporal lobar degeneration
}

Aurélie Pistono ${ }^{1}$, Jérémie Pariente ${ }^{2,3}$ and Mélanie Jucla ${ }^{4}$

${ }^{1}$ Ghent University, Department of Experimental Psychology, Ghent, Belgium

${ }^{2}$ Toulouse NeuroImaging Center, Toulouse University, Inserm, UPS, France

${ }^{3}$ Neurology Department, Neuroscience Centre, Toulouse University Hospital, Toulouse, France

${ }^{4}$ Laboratory of NeuroPsychoLinguistics, University of Toulouse, Toulouse, France.

Corresponding and first author:

Pistono Aurélie

Ghent University, Department of experimental psychology

aurelie.pistono@ugent.be

Henri Dunantlaan, 2

9000 Gent

Belgium 


\begin{abstract}
Disfluencies may reflect various mechanisms: word finding difficulties, planning strategies, interindividual cognitive variability, etc. In the current paper, we examined disfluency production in patients with behavioural variant of Frontotemporal lobar degeneration (bvFTLD), compared to patients with Alzheimer's disease (AD) and healthy older adults. We showed that bvFTLD participants have lower speech rate and produce more incomplete utterances. However, those measures were not correlated with language abilities. On the contrary, AD participants did not differ from healthy controls on disfluency production, but discourse measures were correlated with participants' lexical-semantic impairment. This provides evidence for different disfluency patterns in $\mathrm{AD}$ and FTLD, and a distinct role of disfluencies, depending on the population.
\end{abstract}

Keywords: disfluency, Alzheimer's disease, Frontotemporal lobar degeneration, discourse production

\title{
Introduction
}

Patients with neurodegenerative diseases most often present with discourse impairment compared to healthy older adults (Sharon Ash et al., 2011; Wilson et al., 2010). In the current study, we focused on the behavioural variant of Frontotemporal lobar degeneration (bvFTLD) in comparison with Alzheimer's disease (AD) and. FLTD is an umbrella term for a spectrum of heterogeneous neurodegenerative disorders. Major FLTD phenotypes include bvFTLD (Rascovsky et al., 2011) and primary progressive aphasia (PPA, Gorno-Tempini et al., 2011). The behavioural variant of FTLD involves a deficit of social comportment and personality that often co-occurs with executive function disorders (Rascovsky et al., 2011). Although bvFTLD is classically defined by behavioural and socioemotional changes, impairments often extend to other cognitive functions. In particular, they include early speech and language deficits in around 20\% of patients (Rascovsky et al., 2011). In their 
review of speech and language processing in bvFTLD, Geraudie et al., 2021 showed that bvFTD patients present with extensive but heterogeneous deficits. Domains that were the most consistently impaired were lexical-semantic, reading and writing, as well as prosodic domains. These domains are usually assessed through single word production or comprehension, and few studies focused on connected-speech production. Some studies showed that bvFTLD patients have difficulties in producing coherent, organized discourse, which parallels their difficulties in carrying out organized routine tasks in their daily life (Ash et al. 2006). However, the analysis of other discourse features provided mixed results. For example, some authors concluded that speech rate is globally preserved in bvFTD (e.g., Wilson et al., 2010) while others showed a reduced speech rate, correlated with executive functioning limitations (e.g. Sharon Ash et al., 2011). Similarly, measures of lexicalsemantic abilities in connected speech provide mixed findings, in particular when analysing semantic paraphasia and word-finding difficulties (Geraudie et al., 2021). So far, no study focused on measures that are specific of discourse production, such as disfluency phenomena, in this population. Disfluencies are defined as "phenomena that interrupt the flow of speech and do not add propositional content to an utterance" (Fox Tree, 1995). Even though there is a consensus that disfluencies are most likely multi-factorial, several disfluency phenomena occur when the speaker faces difficulties in language production (e.g. in creating a message (Schnadt \& Corley, 2006) or lexical selection (Pistono \& Hartsuiker, 2021)).

While the language profile is heterogeneous in bvFTLD, lexical-semantic impairment is one of the earliest hallmarks of AD (as evidenced with fluency or naming tasks, Taler \& Phillips, 2008). More and more studies have been focusing on connected-speech production in AD. They show a decline in discourse coherence (e.g., Brandao et al., 2013; Pistono et al., 2018), a reduced lexical content ( e.g., Pistono et al., 2021) and an increase of disfluency production, attributed to word-finding difficulties (e.g. increased use of repetitions and self-corrections in a picture description, de Lira et al., 2011). However, only Ash et al. (2007) analysed AD patients' difficulties during a picture-based 
narrative in relation to their cognitive abilities. They highlighted a negative correlation between wordfinding difficulties and lexical tasks. But the authors did not specify which phenomena were included within word-finding difficulties. On the contrary, when analysing autobiographical narratives, our group observed a positive correlation between early AD participants' pause frequency and their memory capacities (Pistono et al., 2019; Pistono et al., 2016). This suggests that pauses reflect a compensatory mechanism in early $\mathrm{AD}$, used to improve memory retrieval during an autobiographical narrative. In other words, the mechanisms underlying disfluency production in AD may vary with discourse type. However, other disfluency phenomena need to be analysed together with language performance to conclude on the multifactorial role of disfluency in AD. More importantly, it is crucial to stress disfluencies that truly reflect patients lexical-semantic impairment during discourse production.

The current study therefore aims at analysing disfluency production in early bvFTLD compared to early $\mathrm{AD}$ and healthy older adults, during a picture-based narrative. Indeed, analysing these two populations will help unravel the different causes underlying disfluency production. If, in this type of task, disfluency reflects word-finding difficulties in $\mathrm{AD}$, these participants will produce more disfluencies than healthy controls (more self-corrections and repetitions in particular, as in de Lira et al., 2011), and these markers will be correlated with poorer lexical-semantic abilities. In bvFTLD, we predict lower speech rate (as in Ash et al., 2011) and more incomplete sentences, a disfluency that could reflect planning and inhibition difficulties. However, given the heterogeneity of current literature about connected-speech production in bvFTLD, we do not have predictions as to whether other disfluencies will be correlated with language performance in this group.

\section{Methods}

\section{Participants}


Participants were right-handed and native French speakers. They provided written, informed consent before participating in the study. Individuals with early AD were included based on the IWG- 2 criteria (Dubois et al., 2014). Early bvFTLD participants were recruited based on the criteria for the diagnosis of bvFTLD (Rascovsky et al., 2011). Matched healthy control participants were included if they had no memory complaint, no history of neurological or psychiatric disease, and a MMSE (Folstein et al., $1975) \geq 27$. This study was approved by the local ethics committee of Toulouse University Hospital.

\section{Language tasks}

Language was assessed with the GREMOTs battery (Bézy et al., 2016). GREMOTs is a computerized battery of language tests that evaluates both oral and written language as well as production and comprehension at different levels. This battery includes a narrative task (Figure 1), which we analysed more specifically. With regards to the procedure for this task, participants were given the same instructions: "This is a story depicted in 5 pictures. Tell me the story with as many details as possible." During the task, the experimenter remained neutral and avoided speaking in order to ensure uniform conditions for discourse production.

The following variables were analysed:

- Speech rate (number of words/total discourse duration in seconds);

- Proportion of self-corrections: number of self-corrections normalized per 100 words (i.e. when the speaker stops and resumes with a substitution for a word or a new utterance);

- Proportion of repetitions: number of repetitions (of sounds, syllables, words or partial phrases) normalized per 100 words;

- Proportion of filled pauses: number of filled pauses normalized per 100 words;

- Semantic shifts, normalized per 100 words (i.e. abrupt stop within an utterance, after which a new concept begins (Marini et al., 2005)). 


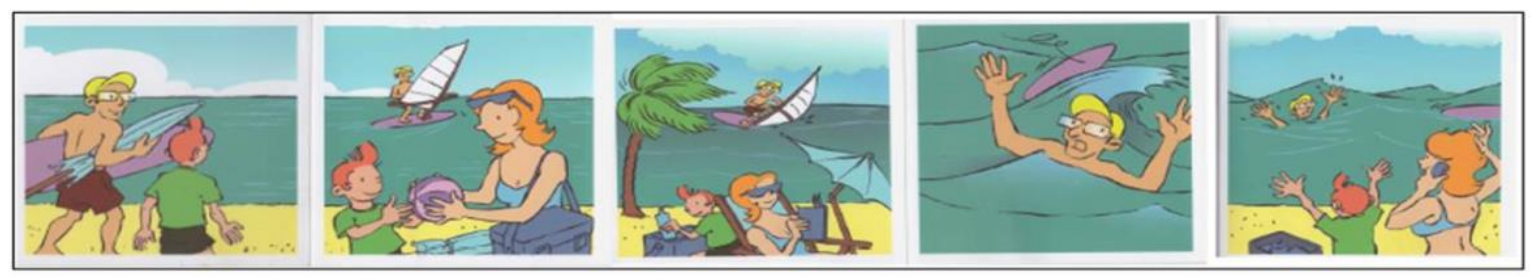

Figure 1. Picture-based narrative from the GREMOTs battery.

\section{Statistical analyses}

Twelve bvFTLD participants, 15 AD participants, and 15 healthy controls (HC) were recruited. Because of the small sample size, inter-groups comparisons were performed with permutation tests in R (version 3.6.1). Kruskal Wallis tests were performed with the coin package (Zeileis et al., 2008), using 10000 permutations. Pairwise comparisons were then conducted using the same method. In a second step, we conducted Kendall correlations between language tests and disfluency, for each group separately.

\section{Results}

The three groups were matched for age, gender and level of education (Table 1). Both AD and bvFTLD groups had lower performance compared to HC during fluency and naming tasks. FTLD patients had lower semantic and phonemic fluency performance than AD patients. Syntactic and phonological performance did not significantly differ between groups after corrections for multiple comparisons.

Table 1. Inter-group differences regarding socio-demographic variables and language assessment. Results that were significant after Bonferroni-Holm corrections are in bold. LoE 1 corresponds to primary school certificate level; LoE 2 to Youth Training or high school diploma; LoE 3 to Baccalaureate levels and higher diplomas.

\begin{tabular}{llllll}
\hline & FTLD & AD & HC & p value & post-hoc tests \\
\hline Age & $65.8 \pm 6.3$ & $67.9 \pm 4.8$ & $67.7 \pm 4.6$ & ns & ns \\
\hline
\end{tabular}




\begin{tabular}{llllll}
\hline Sex & 4 women & 8 women & 7 women & ns & ns \\
\hline Level of Education (LoE) & 4 LoE 2 & 5 LoE 2 & 6 LoE 2 & $n s$ & ns \\
& 8 LoE 3 & 10 LoE 3 & 9 LoE 3 & & \\
\hline MMSE (/30) & $25.3 \pm 3$ & $24.7 \pm 3.1$ & $28.3 \pm 1.3$ & $\mathbf{0 . 0 0 1}$ & HC > AD = FTLD \\
\hline Semantic fluency (fruit) & $9.2 \pm 3.6$ & $14.3 \pm 6.7$ & $19.9 \pm 4.7$ & $<\mathbf{0 . 0 0 0 1}$ & HC > AD > FTLD \\
& & & & & \\
\hline Phonemic fluency (letter V) & $7.6 \pm 5.9$ & $16.3 \pm 7$ & $18.8 \pm 7.5$ & $<\mathbf{0 . 0 0 0 1}$ & HC = AD > FTLD \\
\hline Object naming (/36) & $29.1 \pm 6$ & $32.1 \pm 2.9$ & $34.3 \pm 1.3$ & $\mathbf{0 . 0 0 8}$ & HC > AD = FTLD \\
\hline Action naming (/36) & $29.1 \pm 2.4$ & $30.5 \pm 3.4$ & $33.1 \pm 2.7$ & $\mathbf{0 . 0 0 1}$ & HC > AD = FTLD \\
\hline Famous faces naming (/10) & $5.5 \pm 2.3$ & $4.7 \pm 2.7$ & $8.3 \pm 2.2$ & $<\mathbf{0 . 0 0 0 1}$ & HC > AD = FTLD \\
\hline Word spelling (/12) & $10 \pm 2.4$ & $10.3 \pm 2.2$ & $11.3 \pm 0.9$ & $\mathrm{~ns}$ & $\mathrm{~ns}$ \\
\hline Repetition words (/10) & $9.18 \pm 1.4$ & $9.6 \pm 1$ & $9.9 \pm 0.4$ & $\mathrm{~ns}$ & $\mathrm{~ns}$ \\
\hline Reading words (/30) & $28 \pm 2.9$ & $29.3 \pm 0.8$ & $29.5 \pm 0.6$ & $\mathrm{~ns}$ & $\mathrm{~ns}$ \\
\hline Syntactic comprehension (/24) & $18.8 \pm 5.4$ & $18.9 \pm 3.3$ & $21.6 \pm 2.2$ & $\mathbf{0 . 0 4}$ & $\mathrm{HC}>$ AD = FTLD \\
\hline Sentence spelling (/27) & $24.4 \pm 2.2$ & $24.8 \pm 1.8$ & $25.1 \pm 2.2$ & $\mathrm{~ns}$ & $\mathrm{~ns}$ \\
\hline Reading pseudo-words (/15) & $14.1 \pm 1.5$ & $13.8 \pm 1.1$ & $14.6 \pm 0.7$ & $\mathrm{~ns}$ & $\mathrm{~ns}$ \\
\hline
\end{tabular}

\section{Inter-group comparisons during connected-speech production}

The 3 groups did not significantly differ in terms of discourse length (in number of words, bvFTLD: 97.5 \pm 46.8 ; AD: $109.3 \pm 48.1$; HC: $135.1 \pm 110.8$ ), but the two groups of patients had lower speech rate than healthy controls. Additionally, bvFTLD participants produced significantly more semantic shifts during their narratives (see Table 2).

Table 2. Inter-group differences regarding discourse fluency.

\begin{tabular}{llllll}
\hline & FTLD & AD & HC & p value & Post-hoc tests \\
\hline $\begin{array}{l}\text { Speech rate } \\
\text { (words/sec) }\end{array}$ & $1.7 \pm 0.5$ & $1.8 \pm 0.4$ & $2.4 \pm 0.8$ & $<\mathbf{0 . 0 1}$ & HC > AD = FTLD \\
\hline Repetitions & & & & & \\
\hline Filled pauses & $3.7 \pm 4.5$ & $2.8 \pm 3$ & $2.3 \pm 3.3$ & $\mathrm{~ns}$ & \\
\hline Self-corrections & $1.8 \pm 1.8$ & $2.7 \pm 1.8$ & $2.2 \pm 1.4$ & $\mathrm{~ns}$ & \\
\hline Semantic shifts & $1.9 \pm 1.8$ & $0.9 \pm 1.2$ & $0.5 \pm 0.9$ & $<\mathbf{0 . 0 5}$ & FTLD > HC \\
\hline
\end{tabular}




\section{Intra-group correlations}

Correlations were performed with the most discriminant lexical-semantic tasks: semantic fluency, phonemic fluency, action naming and famous faces naming. In the $\mathrm{HC}$ and bvFTLD group, language performance was not correlated with disfluency production. In the AD group, self-corrections were negatively correlated with famous faces naming ( $\mathrm{r}=-0.52, \mathrm{p}<0.01$, Figure 2$)$, better naming performance were observed in patient with fewer self-corrections. Moreover, speech rate was positively correlated with semantic fluency $(r=0.44, p<0.05$, Figure 2$)$, AD patients with more fluent discourse were also more fluent in semantic driven lexical access.
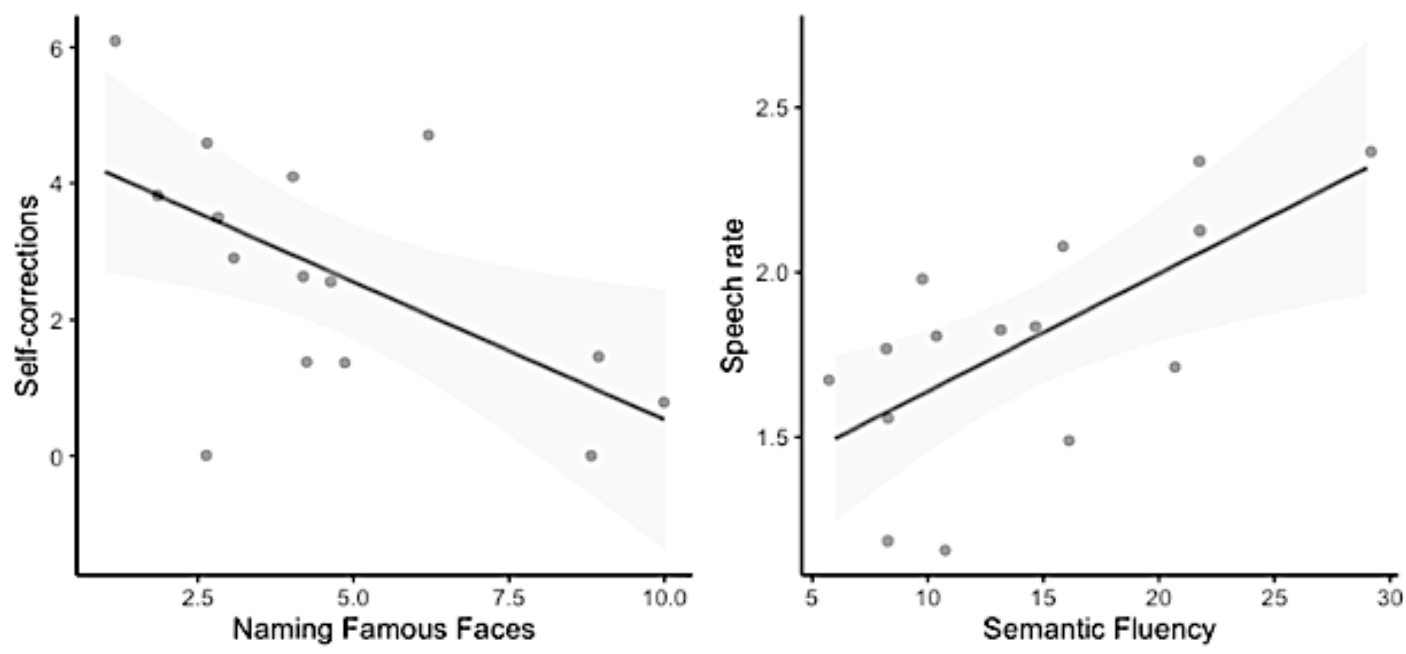

Figure 2. Correlations between lexical tasks and discourse fluency in the AD group.

\section{Discussion}

\section{Language assessment}

Both bvFTLD and AD participants had significant impairment during language assessment (GREMOTs battery) compared to healthy older adults. In particular, differences were mostly related to the lexical-semantic domain. Current results are in line with Geraudie and colleagues (2021), who found that word retrieval and semantic knowledge are most frequently impaired in bvFTLD. Regarding syntactic processing, these authors only found decline on measures of sentence comprehension, which is similar to our study (although not significant after corrections). This review 
also reported lower reading and writing abilities in this population, which is not significant in the current study. However, our population present with prodromal disease with mild cognitive decline that could explain such preservation. Nonetheless, current findings contribute to shed a light on language alteration in early bvFLTD.

AD participants also presented with lexical-semantic decline, which is typically found in early AD (Taler \& Phillips, 2008). More precisely, famous face naming appeared to be the most impaired task in early $\mathrm{AD}$, while phonemic and semantic fluency tasks seem more discriminant for early bvFTLD. By using a language battery dedicated to neurogenerative diseases at an early stage, the current study showed that both groups significantly differ from healthy older adults.

\section{Discourse production}

During discourse production, the three groups were comparable in terms of discourse length, measured in number of words. However, both bvFTLD and AD participants exhibited reduced speech rate, as shown by Ash et al., 2011 and Pistono et al., 2019. bvFTLD participants also produced more semantic shifts. While a previous longitudinal study (Blair et al., 2007) showed that bvFTLD patients have a faster decline in language processing in contrast to the gradual decline found in AD patients, current findings stress features that are discriminant at early stages of AD and bvFTLD. Indeed, current results show that interrupted utterances could be a more specific feature than speech rate to characterize bvFTLD. However, disfluency does not seem related to language processing in this group. Given the heterogeneity of speech and language impairment usually found in this population, disfluencies and discourse difficulties may have several origins in this group. In particular, semantic shifts are closely related to discourse planning and inhibition, and might not reflect language difficulties but rather discourse organization difficulties.

Similarly to bvFTLD participants, AD participants had lower speech rate. However, they did not differ from $\mathrm{HC}$ on other measures. These results differ from de Lira and colleagues (2011) who found an increase of repetitions and self-corrections in AD. However, their participants had a lower 
MMSE (19 \pm 3.4$)$. It is therefore possible that disfluency significantly increases at later stages of AD. Additionally, self-corrections can be seen as evidence that some abilities remain. Indeed, they are the result of a relatively late process of verbal self-monitoring, which is the cognitive system that inspects the speech plan and overt speech and initiates corrections when necessary (Hartsuiker \& Kolk, 2001). It therefore means that early AD participants are still able to correct themselves, while an impaired monitoring system would lead to uncorrected errors. Despite this lack of significant inter-group differences, self-corrections and speech rate were correlated with lexical-semantic tasks in this group, unlike HC. In that sense, current results are similar to our previous investigation of pauses in early AD (Pistono et al., 2019): the proportion of phenomena under study does not differ from HC, but the underlying mechanisms probably do. More precisely, self-corrections could be related to wordfinding difficulties in the $\mathrm{AD}$ group, but not in the $\mathrm{HC}$ group. In other words, lexical-semantic impairment probably leads to errors (and error corrections) in AD, while self-corrections have other underlying causes in healthy aging.

\section{Implications}

This preliminary study represents a first investigation of disfluencies in bvFTLD and AD, that will be further supported by the inclusion of more participants. Additionally, future work is required to analyse disfluency production together with executive functioning in bvFTLD. Indeed, several studies insisted on the role of executive function on discourse production, including disfluency production (Engelhardt, McMullon, \& Corley 2018; Engelhardt et al., 2010). While the current study focused on the links between discourse production and language performance, links with executive function need to be further investigated to disentangle the different causes leading to disfluency in bvFTLD.

More generally, current findings have implications regarding the classification of disfluency phenomena. Indeed, semantic shifts are sometimes grouped with other self-corrections (e.g. Pistono and Hartsuiker, 2021). On the contrary, current study shows that interrupted utterances are hallmarks 
of bvFTLD while other self-corrections are better features of $\mathrm{AD}$ and word-finding difficulties. Further work is now required to develop interventions targeting each population more specifically.

\section{Acknowledgements}

The authors would like to thank the patients and control participants who participated in the study, as well as the promoter of the study, Toulouse University Hospital (CHU).

\section{References}

Ash, S., Moore, P., Antani, S., McCawley, G., Work, M., \& Grossman, M. (2006). Trying to tell a tale: Discourse impairments in progressive aphasia and frontotemporal dementia. Neurology, 66(9), 1405-1413. https://doi.org/10.1212/01.wnl.0000210435.72614.38

Ash, Sharon, Avants, B., \& Grossman, M. (2011). Non-Fluent Speech in Frontotemporal Lobar Degeneration. J Neurolinguistics, 22(4), 370-383. https://doi.org/10.1016/j.jneuroling.2008.12.001.Non-Fluent

Ash, Sharon, Moore, P., Vesely, L., \& Grossman, M. (2007). The decline of narrative discourse in Alzheimer's disease. Brain and Language, 103(1-2), 181-182. https://doi.org/10.1016/j.bandl.2007.07.105

Bézy, C., Renard, A., \& Pariente, J. (2016). GREMOTS Batterie d'évaluation des troubles du langage dans les maladies neurodégénératives. De Boeck supérieur.

Blair, M., Marczinski, C. A., Davis-Faroque, N., \& Kertesz, A. (2007). A longitudinal study of language decline in Alzheimer's disease and frontotemporal dementia. Journal of the International Neuropsychological Society, 13(2), 237-245. https://doi.org/10.1017/S1355617707070269

Brandao, L., Lima, T. M., Pimenta Parente, M., \& Pena-Casanova, J. (2013). Discourse Coherence and its Relation with Cognition in Alzheimer's Disease. Psicologia Em Pesquisa, 7(1), 99107. https://doi.org/10.5327/Z1982-1247201300010011 
de Lira, J O, Ortiz, K. Z., Campanha, A. C., Bertolucci, P. H. F., \& Minett, T. S. C. (2011). Microlinguistic aspects of the oral narrative in patients with Alzheimer's disease. Int Psychogeriatr, 23. https://doi.org/10.1017/S1041610210001092

de Lira, Juliana Onofre, Ortiz, K. Z., Campanha, A. C., Bertolucci, P. H. F., \& Minett, T. S. C. (2011). Microlinguistic aspects of the oral narrative in patients with Alzheimer's disease. International Psychogeriatrics / IPA, 23(3), 404-412. https://doi.org/10.1017/S1041610210001092

Dubois, B., Feldman, H. H., Jacova, C., Hampel, H., Molinuevo, J. L., Blennow, K., DeKosky, S. T., Gauthier, S., Selkoe, D., Bateman, R., Cappa, S., Crutch, S., Engelborghs, S., Frisoni, G. B., Fox, N. C., Galasko, D., Habert, M.-O., Jicha, G. a, Nordberg, A., ... Cummings, J. L. (2014). Advancing research diagnostic criteria for Alzheimer's disease: the IWG-2 criteria. Lancet Neurology, 13(6), 614-629. https://doi.org/10.1016/S1474-4422(14)70090-0

Engelhardt, P. E., McMullon, M.E.G., \& Corley, M. (2018). Individual differences in the production of disfluency: a latent variable analysis of memory ability and verbal intelligence Paul. Quarterly Journal of Experimental Psychology, May, 1-56. https://doi.org/10.1177/1747021818778752

Engelhardt, P. E., Corley, M., Nigg, J. T., \& Ferreira, F. (2010). The role of inhibition in the production of disfluencies. Memory and Cognition, 38(5), 617-628. https://doi.org/10.3758/MC.38.5.617

Folstein, M. F., Folstein, S. E., \& McHugh, P. R. (1975). "Mini-mental state": A practical method for grading the cognitive state of patients for the clinician. Journal of Psychiatric Research, 12, 189-198. https://doi.org/10.1016/0022-3956(75)90026-6

Fox Tree, J. E. (1995). The effects of false starts and repetitions on the processing of subsequent words in spontaneous speech. In Journal of Memory and Language (Vol. 34, Issue 6, pp. 709738). https://doi.org/10.1006/jmla.1995.1032

Geraudie, A., Battista, P., García, A. M., Allen, I. E., Miller, Z. A., Gorno-Tempini, M. L., \& 
Montembeault, M. (2021). Speech and language impairments in behavioral variant frontotemporal dementia: A systematic review. Neuroscience \& Biobehavioral Reviews, 131(July), 1076-1095. https://doi.org/10.1016/j.neubiorev.2021.10.015

Gorno-Tempini, M. L. L., Hillis, a E. E., Weintraub, S., Kertesz, a, Mendez, M., Cappa, S. F. F., Ogar, J. M. M., Rohrer, J. D. D., Black, S., Boeve, B. F. F., Manes, F., Dronkers, N. F. F., Vandenberghe, R., Rascovsky, K., Patterson, K., Miller, B. L. L., Knopman, D. S. S., Hodges, J. R. R., Mesulam, M. M. M., \& Grossman, M. (2011). Classification of primary progressive aphasia and its variants. Neurology, 76, 1-10.

Hartsuiker, R. J., \& Kolk, H. H. (2001). Error monitoring in speech production: a computational test of the perceptual loop theory. Cognitive Psychology, 42(2), 113-157. https://doi.org/10.1006/cogp.2000.0744

Marini, A., Boewe, A., Caltagirone, C., \& Carlomagno, S. (2005). Age-related differences in the production of textual descriptions. Journal of Psycholinguistic Research, 34(5), 439-463. https://doi.org/10.1007/s10936-005-6203-z

Pistono, A., Jucla, M., Barbeau, E. J., Saint-Aubert, L., Lemesle, B., Calvet, B., Köpke, B., Puel, M., \& Pariente, J. (2016). Pauses during Autobiographical Discourse Reflect Episodic Memory Processes in Early Alzheimer's Disease. Journal of Alzheimer's Disease, 50(3), 687698. https://doi.org/10.3233/JAD-150408

Pistono, A., Pariente, J., Bézy, C., Lemesle, B., Le Men, J., \& Jucla, M. (2019). What happens when nothing happens? An investigation of pauses as a compensatory mechanism in early Alzheimer's disease. Neuropsychologia, 124, 133-143. https://doi.org/10.1016/J.NEUROPSYCHOLOGIA.2018.12.018

Pistono, Aurélie, \& Hartsuiker, R. (2021). Eye-movements can help disentangle mechanisms underlying disfluency. Language, Cognition and Neuroscience, $0(0), 1-18$. https://doi.org/10.31234/osf.io/6mx2y

Pistono, Aurélie, Jucla, M., \& Pariente, J. (2018). Discourse macrolinguistic impairment as a 
marker of linguistic and extralinguistic functions decline in early Alzheimer's disease. International Journal of Language \& Communication Disorders, 54(3), 390-400. https://doi.org/10.1111/1460-6984.12444

Pistono, Aurélie, Senoussi, M., Guerrier, L., Rafiq, M., Giméno, M., Péran, P., Jucla, M., \& Pariente, J. (2021). Language Network Connectivity Increases in Early Alzheimer's Disease. Journal of Alzheimer's Disease, 82(1), 447-460. https://doi.org/10.3233/jad-201584

Rascovsky, K., Hodges, J. R., Knopman, D., Mendez, M. F., Kramer, J. H., Neuhaus, J., Van Swieten, J. C., Seelaar, H., Dopper, E. G. P., Onyike, C. U., Hillis, A. E., Josephs, K. A., Boeve, B. F., Kertesz, A., Seeley, W. W., Rankin, K. P., Johnson, J. K., Gorno-Tempini, M. L., Rosen, H., ... Miller, B. L. (2011). Sensitivity of revised diagnostic criteria for the behavioural variant of frontotemporal dementia. Brain, 134(9), 2456-2477. https://doi.org/10.1093/brain/awr179

Schnadt, M. J., \& Corley, M. (2006). The influence of lexical, conceptual and planning based factors on disfluency production. 28th Annual Conference of the ..., 8-13.

Taler, V., \& Phillips, N. A. (2008). Language performance in Alzheimer's disease and mild cognitive impairment: a comparative review. Journal of Clinical and Experimental Neuropsychology, 30(5), 501-556. https://doi.org/10.1080/13803390701550128

Wilson, S. M., Henry, M. L., Besbris, M., Ogar, J. M., Dronkers, N. F., Jarrold, W., Miller, B. L., \& Gorno-Tempini, M. L. (2010). Connected speech production in three variants of primary progressive aphasia. Brain : A Journal of Neurology, 133(Pt 7), 2069-2088. https://doi.org/10.1093/brain/awq129

Zeileis, A., Hornik, K., Wiel, M. A., \& Hothorn, T. (2008). Implementing a class of permutation tests: The coin package. Journal of Statistical Software, 28(8). http://epub.wu.ac.at/4004/\%5Cnpapers3://publication/uuid/BF1BBE55-2A35-44D0-96DAFCCC209A5334 
